\title{
Fusion protein tTF-EG3287 induces occlusion of tumor vessels and impairs tumor growth in human colon cancer
}

\author{
G. Q. QIU ${ }^{1, *}, X . X E^{1, *}$, B. ZHAO ${ }^{2}$, L. Z. XU' ${ }^{1}$, Y. Q. CHEN ${ }^{1, *}$ \\ ${ }^{1}$ Department of Oncology, Chenggong Hospital Affiliated to Xiamen University, Xiamen University, Xiamen, Fujian 361000 China; ${ }^{2}$ Medical \\ College, Xiamen University, Xiamen, Fujian 361002, China \\ *Correspondence: chenyq707@163.com \\ \#Contributed equally to this work.
}

Received July 22, 2018 / Accepted October 22, 2018

\begin{abstract}
The problems including narrow indications, low drug loading, and difficulty in intervention severely affect the clinical efficacy of anti-tumor embolization. Here, we designed a novel tTF-EG3287 protein consisting of the truncated tissue factor (tTF) fused with the bicyclic polypeptide which was encoded by exons 7 and 8 for accurate localization in the tumor vascular endothelial cells (EG3287). This study aims to explore its anti-cancer effect. Gene sequencing was used to verify the fusion gene and SDS-PAGE gel to confirm the optimal induction time and concentration of tTF-EG3287. Nickel affinity chromatography column was used to purify the fusion protein. Confocal microscopy was used to assess the target activity of tTF-EG3287 on colon cancer cells in vitro. Thrombelastography assay was used to identify the pro-coagulant activity of tTF-EG3287. In in vivo experiments, the specific localization of tTF-EG3287 in tumor tissues and the effect of tTF-EG3287 on tumor thrombosis were further detected by in vivo imaging and HE staining, respectively. The tTF-EG3287 fusion protein was efficiently purified by nickel-affinity chromatography column. Moreover, tTF-EG3287 fusion protein showed strong coagulation a ctivity and specific binding ability to the cell surface of colon cancer. In vivo, tTF-EG3287 stably and persistently accumulated in tumor tissues, and specifically induced mixed thrombus formation in tumor vessels, and then impaired tumor growth (tumor inhibition rate $=79.2 \%, \mathrm{p}<0.01$ ). Our data prove that the fusion protein tTF-EG3287 could be used as a novel and promising anti-cancer strategy and has great potential value for clinical applications.
\end{abstract}

Key words: colon cancer, $t$ TF-EG3287, targeted therapy, vascular thrombosis, xenografts

As one of the most frequently diagnosed malignant diseases, colon cancer presents a rising trend in the morbidity and mortality [1-3]. It is estimated that there are about $1,020,000$ newly diagnosed patients in the world every year. Surgical treatment is the preferred treatment for patients with early colon cancer [4]. But for patients with non-resectable metastatic colorectal cancer (mCRC), chemotherapy is the main treatment option [5]. Recently, the emergence of targeted drugs has significantly improved the colon cancer treatment [6]. The combination of targeted drugs with chemotherapeutic drugs significantly increases the therapeutic effect and has a good tolerance in the colon cancer treatment [7]. Therefore, it is of great importance to explore new targeted drugs in the therapy of colon cancer.

The growth and metastasis of tumor cells is closely related to tumor angiogenesis [8]. Many reports have shown that anti-angiogenesis drugs including bevacizumab and cetuximab which target VEGF or its receptors significantly increase the overall survival (OS) of $\operatorname{mCRC}[9,10]$. The tTF is extracellular region of the TF. Reports have demonstrated that tTF can combine with FVII and activate factor X (FX) and activate the extrinsic coagulation pathway $[11,12]$. Kessler, et al. has proven that the fusion of integrin a $5 \beta 1$ with tTF could induce embolization of the tumor blood vessel in adenocarcinoma, melanoma and fibrosarcoma, thereby suppressing the tumor growth [13]. The tTF-NGR designed by Christian, et al. reduced the growth of fibrosarcoma transplanted tumors [14]. As an effector molecule targeting tumor vascular embolism, we need to explore tTF-related targeted drugs for colon cancer treatment. Neuropilin-1 (NRP-1) is closely related to the growth of axons, angiogenesis and tumor metastasis, and also widely expressed in glioma, pancreatic cancer, colon cancer and other solid tumors [15-17]. To determine the role of NRP1 in biological functions, Jia et al. has designed the EG3287 polypeptide which is the binding site of VEGF165 and NRP1. EG3287 can inhibit the cross- 
linking of VEGF165 and KDR when co-expressed with NRP1 in human umbilical vein endothelial cells, thereby having anti-tumor effect [18].

In this study, we optimized induction condition and increased the amount of tTF-EG3287 expression. Besides, the pro-coagulant activity of tTF-EG3287 and its localization in cells/tissues were detected. Moreover, the effect of tTF-EG3287 on tumor growth was also explored in vivo experiments. These data provide evidence that tTF-EG3287 could be used as a novel drug targeting vascular embolization in colon cancer.

\section{Materials and methods}

Recombinant plasmids. The tTF-EG3287 recombinant vectors were constructed and preserved by Cancer Research Center of Medical School, Xiamen University.

Cell culture. Human colon cancer cell lines (HT29 and HUVEC) were purchased from American Type Culture Collection (USA) and cultured in DMEM (Invitrogen-Life technologies, USA) supplemented with $10 \%$ fetal bovine serum (FBS, Sigma-Aldrich, Germany). All cells were maintained in a humidified atmosphere at $37^{\circ} \mathrm{C}$ with $5 \% \mathrm{CO}_{2}$.

Confocal microscopy. The tTF-EG3287 and tTF fusion proteins were labeled with Rhodamine B isothiocyanate (RBITC). For immunofluorescence assay, the HT29 and HUVEC cells $\left(1 \times 10^{5}\right.$ cells $\left./ \mathrm{ml}\right)$ were seeded into a 6 -wellplate with glass coverslips and maintained in a humidified atmosphere at $37^{\circ} \mathrm{C}$ with $5 \% \mathrm{CO}_{2}$ until the cells reached $50-60 \%$ confluence. The supernatant was removed and the coverslips were washed three times with PBS ( $\mathrm{pH} 7.4$ ). Afterwards, $2 \mathrm{ml} 4 \%$ paraformaldehyde was added to cells for fixation. Then the RBITC-labeled tTF-EG3287 and tTF $(20 \mu \mathrm{g})$ were added to the cells, respectively. After $1 \mathrm{~h}$ the cells were washed twice with PBS and then incubated with Hoechst 33258. Images were analyzed with the laser scanning confocal microscope (LEICA, Germany).

The expression and purification of fusion protein. The highest producing clones containing tTF-EG3287 pET32b (+) recombinant plasmid were selected and then cultured in Luria-Bertain medium (OXID, UK) with a final concentration of $50 \mu \mathrm{g} / \mathrm{ml}$ ampicillin (Shenggong, China) for amplification culture. The optimal concentration of isopropylthio- $\beta$ D-galactoside (IPTG, Sigma, USA) inducer was added when the OD600 value reached about $0.6 \sim 0.8$, and the tTF-EG3287 fusion protein was efficiently expressed in the optimal induction time.

For the purification of fusion protein, the bacteria were harvested by centrifuging at $13800 \times \mathrm{g}$ for $15 \mathrm{~min}$ at $4^{\circ} \mathrm{C}$ and homogenized by sonicating in an ice bath. The lysate was then centrifuged at $13800 \times \mathrm{g}$ for $30 \mathrm{~min}$ at $4{ }^{\circ} \mathrm{C}$ to obtain the precipitate. Then, the precipitate was incubated in the solution containing $2.5 \%$ inclusion body overnight at $4{ }^{\circ} \mathrm{C}$ for dissolution and the suspension was obtained after centrifuging at $13800 \times \mathrm{g}$ for $30 \mathrm{~min}$ at $4^{\circ} \mathrm{C}$. The supernatant was filtered through a $0.22 \mu \mathrm{m}$ filter and the fusion proteins were purified and refolded by a Nickel affinity chromatography column (HuiTan Bio, China) according to the manufacturer's instructions. To renature the fusion protein, the suspension was firstly dialyzed in renaturation buffer containing urea concentration gradient ( $6 \mathrm{M}, 4 \mathrm{M}, 2 \mathrm{M}, 1 \mathrm{M})$ which was changed every $8 \mathrm{~h}$, and finally in $0.01 \mathrm{M}$ urea in PBS pH 8.0 buffer for $24 \mathrm{~h}$ to remove the salt. The fusion protein was condensed by centrifugation at $5000 \times \mathrm{g}$ for $60 \mathrm{~min}$ with the use of Amicon ${ }^{\circledR}$ Ultra ultrafiltration centrifuge tube. Finally, the fusion protein was analyzed under denaturation conditions on SDS-PAGE.

Trombelastography assay. Thrombelastography (also known as TEG), which was invented by the German Hartert in 1948, is a method to detect whether the whole blood samples have a normal coagulation function by analyzing physical characteristics of blood clots. All TEG parameters were recorded from standard tracings: reaction time ( $\mathrm{R}$, minutes), alpha angle (angle, degrees), coagulation time ( $\mathrm{K}, \mathrm{min})$, maximum amplitude (MA, mm). Using this method, $300 \mu \mathrm{l}$ of bloods containing $3.8 \%$ sodium citrate (volume ratio 1:9) were added into $20 \mu \mathrm{l}$ of tTF or tTF-EG3287 (which had the same concentration as tTF) and $20 \mu \mathrm{l}$ of $12.5 \mathrm{mM} \mathrm{CaCl}_{2}$ solution. Blood with added PBS only were used as the blank control.

Establishment of tumor transplantation model for colon cancer. All BALB/c nude mice (6-week-old, female) used in this study were obtained from the Animal Center of Xiamen University (Xiamen, China) and were approved by the Ethics Committee of Xiamen University. Briefly, the left and right armpits of nude mice were inoculated with $2 \times 10^{6}$ HT29 cells. When the tumors reached $120 \mathrm{~mm}^{3}$ volume, they were peeled off, cut approximately into $4-8 \mathrm{~mm}^{3}$ pieces and subcutaneously implanted into the right upper limb of nude mice. Tumor growth was observed every 3 days.

Method of administration. Fifteen nude mice with successful subcutaneous transplant were randomly divided into 3 groups: saline group $(n=5)$, tTF-EG3287 group $(n=5)$ and tTF group $(n=5)$. A $50 \mathrm{mg} /$ mouse dose of tTF-EG3287 or tTF was injected into the mice via the tail vein, while saline was used as a negative control. The body weight and tumor size were recorded after each administration. The calculation formula for tumor volume was: $\mathrm{V}=(\text { width } \times \text { length })^{2} / 2$. After 27 days, the nude mice were euthanized, and the tumors were weighed. Tumor suppressor rate (TGI) which was used to evaluate the inhibitory effect of drugs on tumor growth in vivo was calculated using the following formula: $\mathrm{TGI}=(1$ - tumor weight in treatment group/tumor weight in control group) $\times 100$. In this study, saline group was used as the control group, while tTF-EG3287 and tTF group were the experimental groups.

In vivo imaging of nude mice with tumor transplantation for colon cancer. The concentration of thrombin tTF-EG3287 and tTF was prepared in $1 \mathrm{mg} / \mathrm{ml}$ concentration, while fluorescent Cy5.5-labeled proteins were dissolved in DMSO to prepare a solution with concentration of 
$1 \times 10^{4} \mathrm{mg} / \mathrm{ml}$. Then, the two coagulant protein solutions were mixed with the same volume of Cy5.5 solution and firstly placed at $37^{\circ} \mathrm{C}$ constant temperature incubator for lightproof reaction for $2 \mathrm{~h}$, and then moved to $4^{\circ} \mathrm{C}$ for overnight. Afterwards, the proteins tTF-EG3287 and tTF labeled by Cy5.5 were collected by centrifuging at $17250 \times \mathrm{g}$ for $5 \mathrm{~min}$. For in vivo imaging, six nude mice with successful subcutaneous transplants were randomly divided into 3 groups: saline group $(n=2)$, tTF-EG3287 group $(n=2)$ and tTF group $(n=2)$. The nude mice in saline group received $100 \mu$ free Cy5.5 via tail vein injection, while the other two groups received $100 \mu \mathrm{l}$ of tTF-EG3287 and tTF labeled by Cy5.5. The enrichment of fluorescence labeled with tTF-EG3287 and tTF in nude mice and subcutaneous tumors was observed through in vivo imaging within $24 \mathrm{~h}$ after drug administration, the blank nude mice were considered as the background control. Then the mice were euthanized and the brain, heart, liver, spleen, lungs and kidney of nude mice were extracted for further organ imaging.
H\&E staining. To explore the changes of blood vessel thrombus in tumor and normal tissues obtained from tTF-EG3287 group and tTF group, tissues were cut into $1 \mathrm{~cm} \times 1 \mathrm{~cm} \times 0.2 \mathrm{~cm}$ sections and stained with HematoxylinEosin ( $\mathrm{H} \& \mathrm{E}$, Beyotime Institute of Biotechnolgy, China) according to the manufacturer's instructions. The HE stained sections were photographed under a conventional optical microscope.

Statistics. All experiments were repeated at least three times. SPSS19.0 statistical software was used for data analysis. Data were presented as the mean \pm SD. A $p<0.05$ was considered statistically significant.

\section{Results}

Expression and Purification of tTF-EG3287. We firstly used gene sequencing method to ensure the accuracy of tTF-EG3287 recombinant gene sequence (Figures 1A and 1B). SDS-PAGE analysis was used to confirm that the
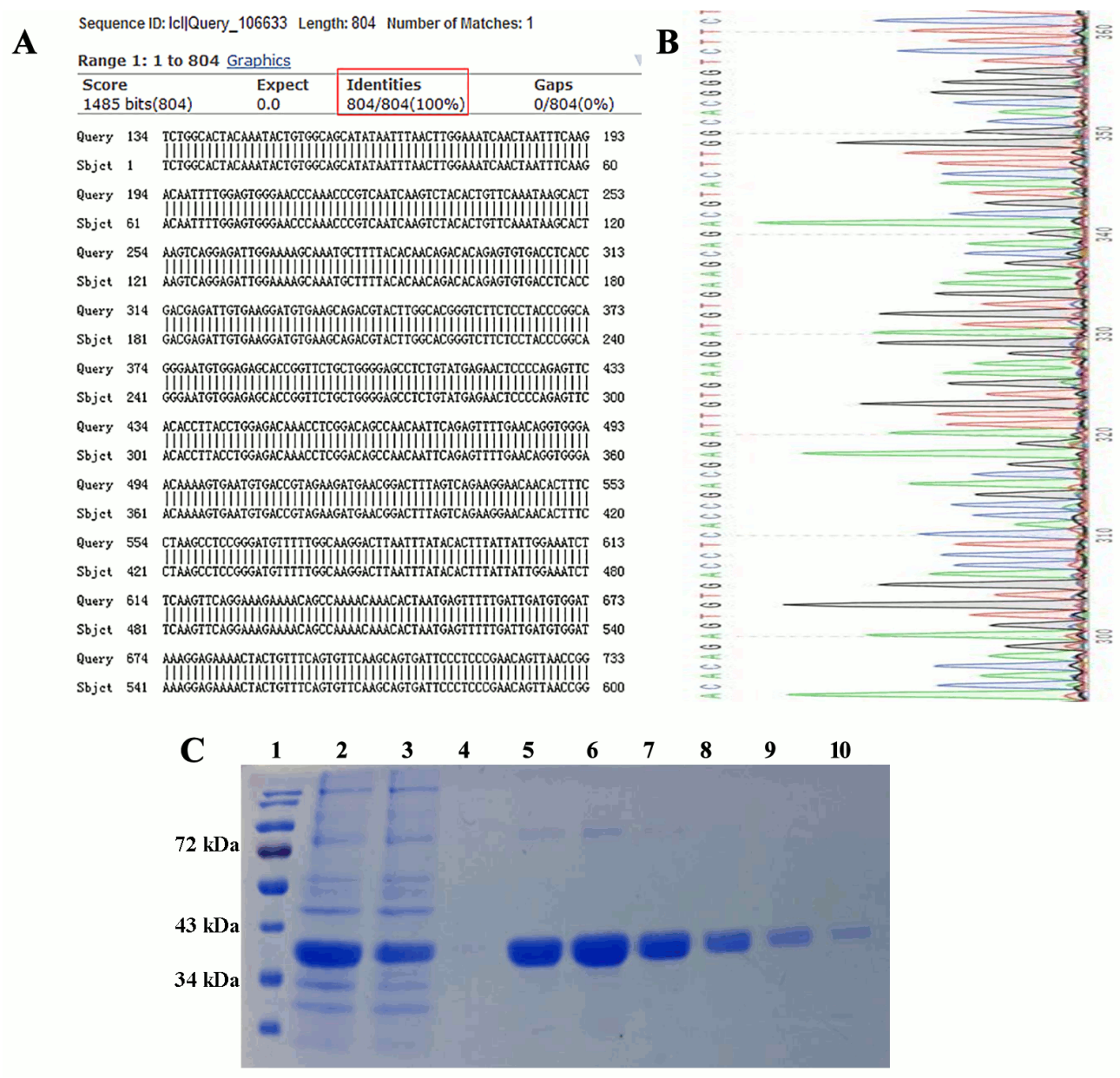

Figure 1. Expression and purification of tTF-EG3287. A) Results of tTF-EG3287 gene sequencing analysis and comparison. B) Part sequencing analysis of tTF-EG3287 recombinant gene. C) Analysis of tTF-EG3287 eluting product by SDS-PAGE. Lane 1, molecular weight markers; Lane 2, total protein from E. coli without induction; Lanes 3-4, Flow-through liquid; Lanes 5-12, Eluting products from Ni-6FF(TED) column. 
optimal induction time was from 7 to $9 \mathrm{~h}$ and the optimal induction concentration was $0.4 \mathrm{mM}$ via detecting the protein levels of tTF-EG3287 which were induced by IPTG at different concentrations and times (Figures S1 and S2). Then, the fusion protein of tTF-EG3287 was expressed by E.coli under optimal induction conditions. After purification by nickel column, the SDS-PAGE electrophoresis analysis of purified proteins showed that there was a single band with the expected size of approximately $35 \mathrm{kDa}$ (Figure 1C). In addition, the final concentration of tTF-EG3287 protein was detected through comparative analysis of different concentrations of BSA and was $2.17 \mathrm{mg} / \mathrm{ml}$ (Figure S3). Moreover, a large number of truncated tissue factors (tTF) was obtained using the same method, providing sufficient material for the subsequent experiments.

Identification of tTF-EG3287 pro-coagulant activity. Thrombus elastography (CFMS) can continuously record the coagulation process of entire blood by measuring the elastic changes associated with fibrinogen polymerization and is currently used in standard coagulation tests. In our study, we found that the tTF-EG3287 and tTF showed similar ability in accelerating the pro-coagulation process when compared to the control group. However, tTF-EG3287 protein showed stronger pro-coagulation effect than tTF protein at the same concentration. Meanwhile, the pro-coagulant effect of these two proteins was enhanced by increasing the concentration.

Target activity of tTF-EG3287 on colon cancer cells in vitro. Studies have shown that NRP1 is highly expressed in HT29 and HUVEC cells [19, 20]. Meanwhile, EG3287 can competitively bind to NRP1 without activating the intracellular downstream signaling pathway [18]. To further explore the target activity of fusion protein on colon cancer cells, HT29 cells and HUVEC cells were incubated with RBITClabeled tTF-EG3287 or tTF, and the binding of tTF-EG3287 or tTF to colon cancer cells was further detected via using the laser scanning confocal microscopy. As shown in Figure 3, there was a strong fluorescence on the membranes and cytoplasms of HT29 and HUVECs treated with tTF-EG3287, but bare fluorescence signal was found on tTF-RBITC-treated cells. These results demonstrate that the tTF-EG3287 might specifically bind to the membrane and further get inside the colon cancer cells, thereby showing anti-tumor effect.

In vivo distribution of tTF-EG3287. To test the biological distribution of drugs in tumor-bearing nude mice, the fusion protein tTF-EG3287 and tTF were labeled with Cy5.5 fluorescent dye and injected through tail vein into nude
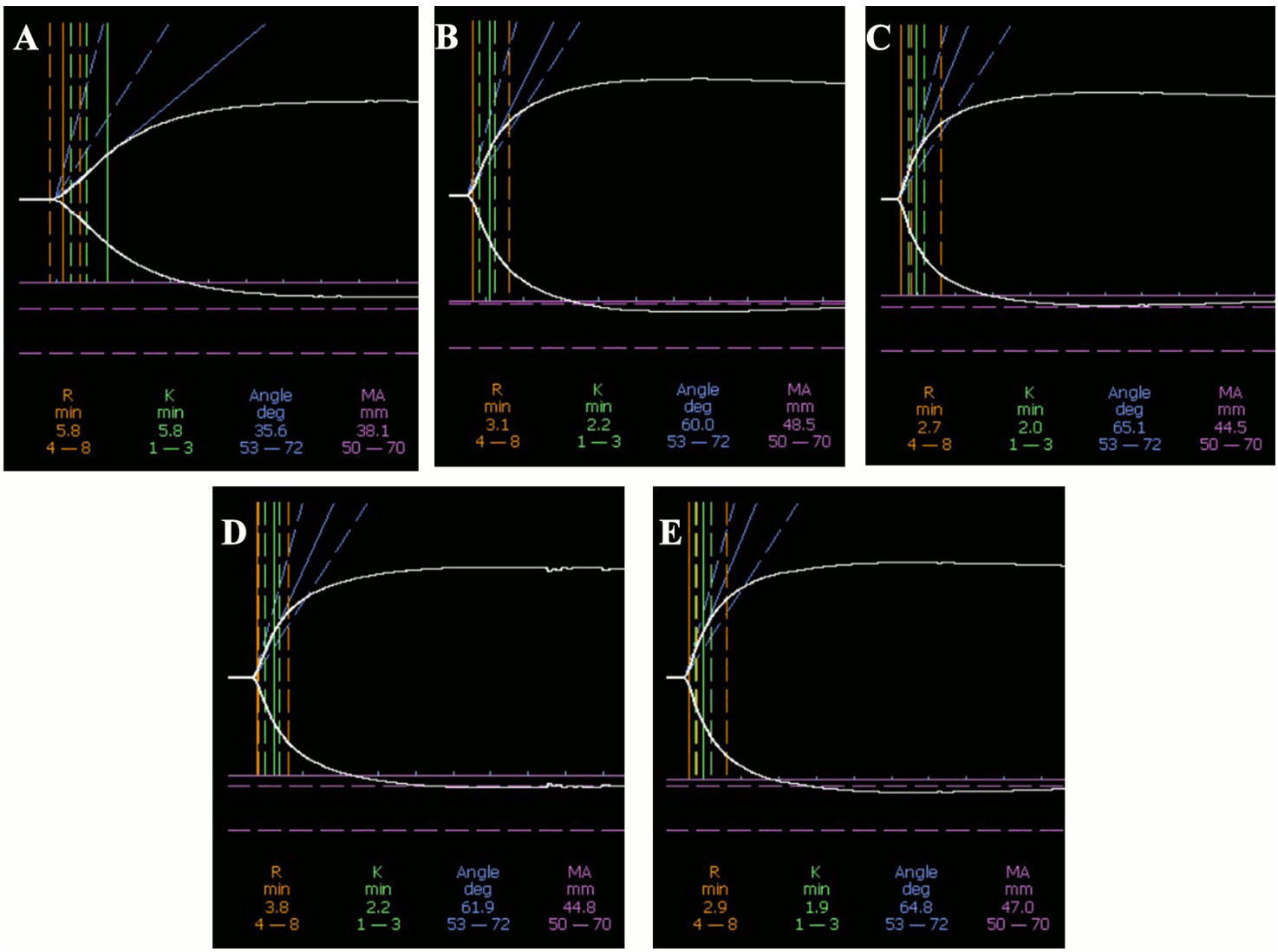

Figure 2. Identification of tTF-EG3287pro-coagulant activity. Evaluation clotting of tTF-EG3287 and tTF proteins by Thrombelastograph Test. A) The blood was treated with $20 \mu \mathrm{l}$ PBS. B) The blood was added into $20 \mu \mathrm{l} \mathrm{of} 0.5 \mathrm{mg} / \mathrm{ml} \mathrm{tTF}$. C) The blood was treated with $20 \mu \mathrm{lof} 1 \mathrm{mg} / \mathrm{ml} \mathrm{tTF}$. D) The blood was added into $20 \mu \mathrm{l} \mathrm{of} 0.5 \mathrm{mg} / \mathrm{ml}$ tTF-EG3287. E) The blood was treated with $20 \mu \mathrm{lof} 1 \mathrm{mg} / \mathrm{ml} \mathrm{tTF-EG3287.}$ 


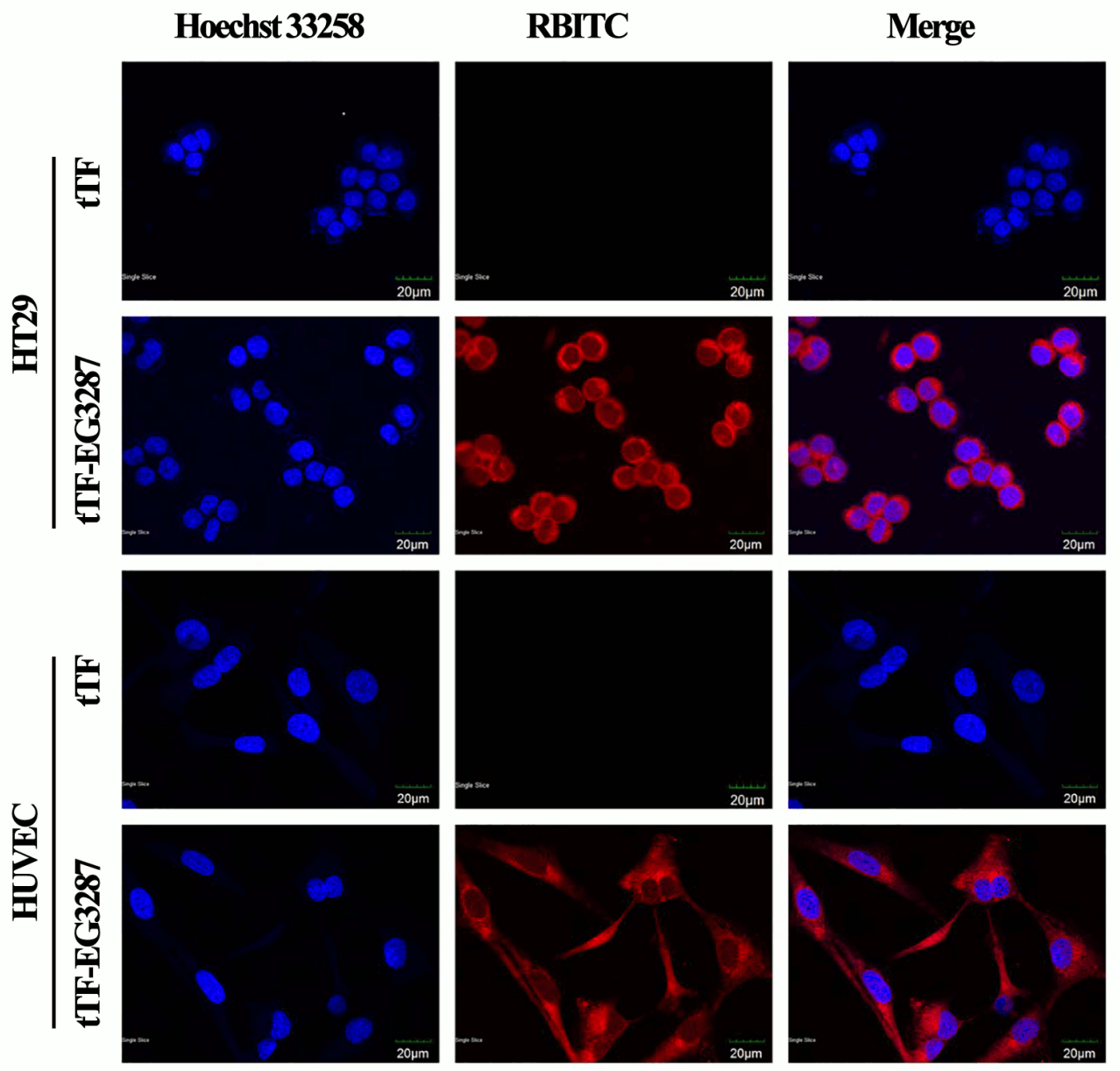

Figure 3. Confocal immunofluorescent analysis of tTF and tTF-EG3287 localization in HT29 and HUVEC cells. HT29 an HUVEC cells were incubated with RBITC-labeled tTF-EG3287 and tTF (red) for $1 \mathrm{~h}$, the cell nuclei were labeled with Hoechst 33258 (blue) $(\mathrm{bar}=20 \mu \mathrm{m})$.

mice bearing colon carcinoma xenotransplants. As shown in Figure 4A, the tTF-EG3287 was gradually enriched in transplanted tumors and organs with abundant blood flow including livers, kidneys and lungs. The fluorescence intensity reached the peak at $8 \mathrm{~h}$ and lasted for $24 \mathrm{~h}$ in tumors. Although the accumulation of tTF-EG3287 was highest in liver, the fluorescence faded after $9 \mathrm{~h}$. The main reason was that liver was the main organ of drug metabolism, therefore the drug levels in liver increased at first, and then gradually decreased with drug metabolism. In kidney, the fluorescence intensity was lowest after $24 \mathrm{~h}$ due to continuous circulation and metabolism of drugs. However, the fluorescence intensity in lung was not attenuated from $1 \mathrm{~h}$ to $24 \mathrm{~h}$, which might be related to the drug toxicity. In the nude mice treated with tTF, the drug was distributed throughout the body with blood flow, but the fluorescence intensity disappeared almost completely after $10 \mathrm{~h}$ for the metabolism of liver and kidney. All these results demonstrate that free tTF had no obvious accumulation in tumors or other normal tissues and organs, but fusion protein tTF-EG3287 was stable and durable targeting the xenograft tumors of colon cancer in nude mice.

To further investigate the distribution and metabolism of fusion protein in nude mice in vivo, the brain, heart, liver, spleen, lungs, kidney and tumor of nude mice were extracted for further organ imaging. Results also proved that the tTF-EG3287 was mainly located in tumor tissues, but without accumulation in other normal tissues and organs (Figure 4B). However, there was little accumulation of tTF in all organs and tumors, further proving that tTF-EG3287 was more tumor-specific than tTF.

The inhibitory effect of tTF-EG3287 protein on the xenograft tumor growth. In order to assess the therapeutic effect of tTF-EG3287 on colon cancer, the nude mice bearing colon carcinoma xenotransplants were injected with tTF-EG3287 and tTF via tail vein. As shown in Figure 5A, the tumor size of the Saline group was similar to that of tTF group, while the tumor size of the tTF-EG3287 treated group was significantly reduced. We also proved that the growth rate of transplanted tumors in tTF-EG3287 treated 

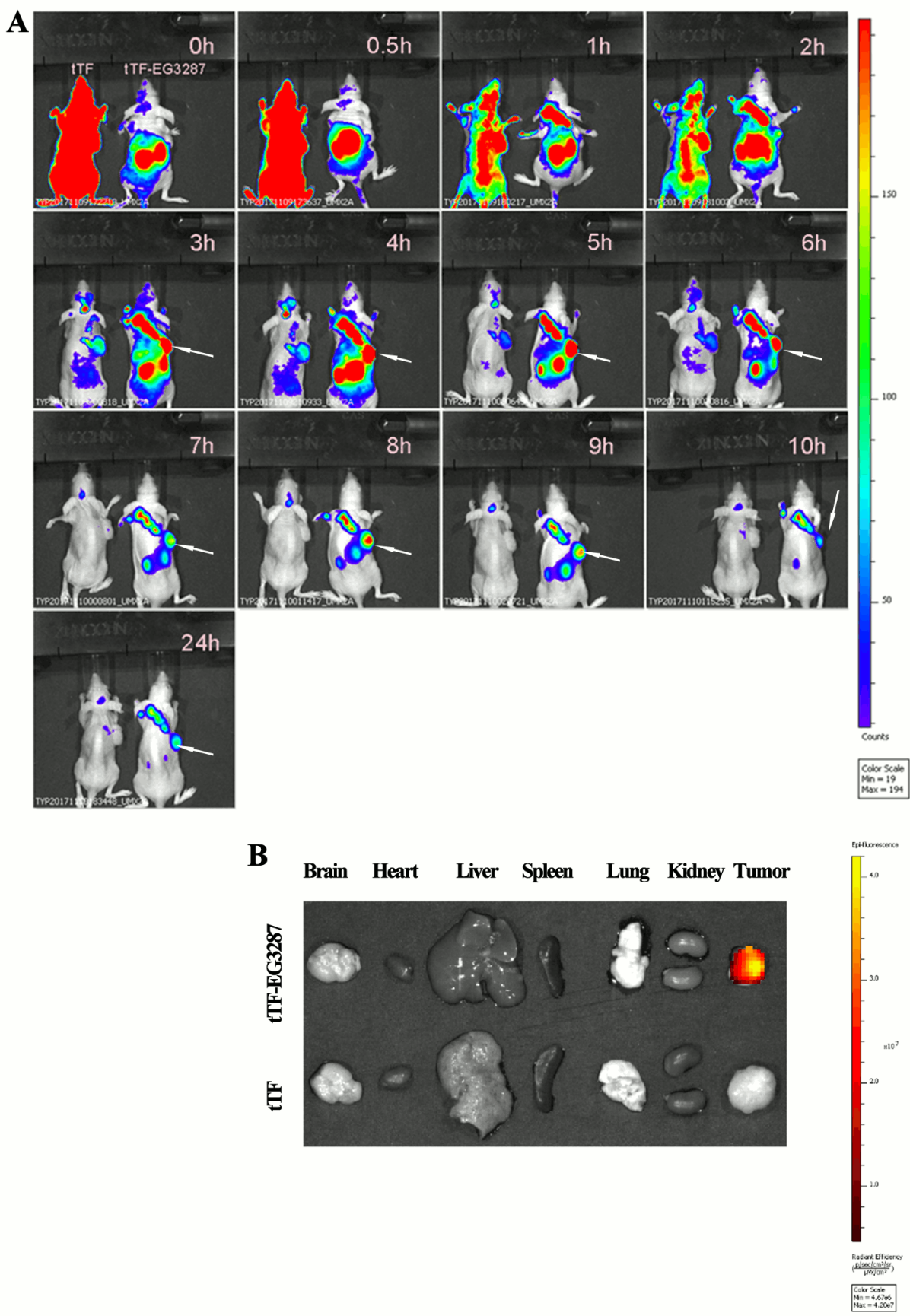

Figure 4. In vivo distribution of tTF-EG3287. A) Fusion proteins tTF-EG3287 and tTF labeled with Cy5.5 fluorescent dye were injected into nude mice bearing colorectal carcinoma xenotransplants through tail vein. Selective accumulation of Cy5.5-labeled tTF-EG3287 and tTF in nude mice was explored by an in vivo imaging system after injection at $0,0.5,1,2,3,4,5,6,7,8,9,10$, and $24 \mathrm{~h}$. Arrows represent the transplanted tumors. B) Optical in vivo imaging in different organs using Cy5.5-labeled tTF and tTF-EG3287.

group was significantly inhibited when compared to the control group during continuous treatment between 12 and 27 days. However, there was no significant difference in tumor volume between $\mathrm{tTF}$ treated group and control group (Figure 5B, $\mathrm{p}<0.01$ ). We also demonstrated that tumors in tTF-EG3287 treated group were significantly smaller than in the control group after 27 days of treatment (Figure 5C). In addition, we also proved that the tumor inhibition rate of tTF was as high as $79.2 \%$ (Figure 5D, $\mathrm{p}<0.01$ ). Therefore, fusion protein tTF-EG3287 had a significant anti-tumor effect, while protein $\mathrm{tTF}$ had no statistical significance in the treatment of transplanted tumors. 
Effect of tTF-EG3287 on tumor thrombosis. Tumor thrombus formation capacity of tTF-EG3287 was tested in nude mice bearing colon carcinoma xenotransplants by $\mathrm{H} \& \mathrm{E}$ staining. After treatment with tTF-EG3287 or tTF for $24 \mathrm{~h}$, the thrombosis in the blood vessels of tumors treated with tTF-EG3287 was formed extensively, but not in those treated with tTF. However, we also found ischemic necrotic foci located in liver and lung treated with tTF-EG3287. In addition, there was no thrombosis in blood vessels of all organs extracted from nude mice after tTF treatment.

\section{Discussion}

At present, surgery and chemotherapy are main treatment modalities used in colon cancer patients. However, chemical drugs often cause damage to normal cells while killing the cancer cells [21]. Meanwhile, tumors can develop multidrug resistance to chemotherapeutic drugs through a variety of mechanisms [22]. Therefore, it is urgent to find a new way to treat colon cancer.

Tumor blood vessels are the basis of growth, invasion and metastasis of solid tumor [23]. Recently, many fusion proteins have been prepared by the combination of antitumor vascular antibodies and tTF with specific transmission function of antibodies and potential coagulation function of tTF $[24,25]$. Reports have shown that the specific tumor embolization effect of tTF fusion protein depends on the vector specificity and the expression and distribution of its antigen or receptor. NRP-1, as the co-receptor of VEGF165, is upregulated on the surface of tumor neovascularization endothelial cells and rarely expressed in normal tissues [26, 27]. Therefore, EG3287, a short peptide that can specifically bind to NRP-1, was used as the carrier of tumor vascular targeted drugs in this study. In addition, although tTF retains the ability to activate FX, it is still significantly lower that of the complete trans-membrane $\operatorname{TF}[24,25]$. This is because the TF-VIIa complex requires a negatively charged phospholipid membrane to effectively bind and activate FX. In this study, the EG3287 part of tTF-EG3287 fusion protein not only provided specific transfer function, but also played similar function as TF membrane structure after binding to the membrane receptor. Therefore, the exogenous coagulation pathway was activated by tTF only when the EG3287 part of the tTF-EG3287 fusion protein binds to the surface of tumor vascular cell membrane. Here, we proved that the fusion protein tTF-EG3287 mainly locates on the surface of colon cancer cells and tumor tissue vessels, indicating that tTF-EG3287 fusion protein not only retains part of the biological activity, but also specific bond to tumor vascular endothelial cells. Further histological analysis also revealed that large area of embolism was produced in tumor vessels treated with tTF-EG3287, but no thrombosis was found in the vessels of other normal tissues.

In vivo experiments showed that although the distribution of tTF-EG3287 in tumors was stable and durable, fusion protein tTF-EG3287 had obvious toxic side effects on lung
A
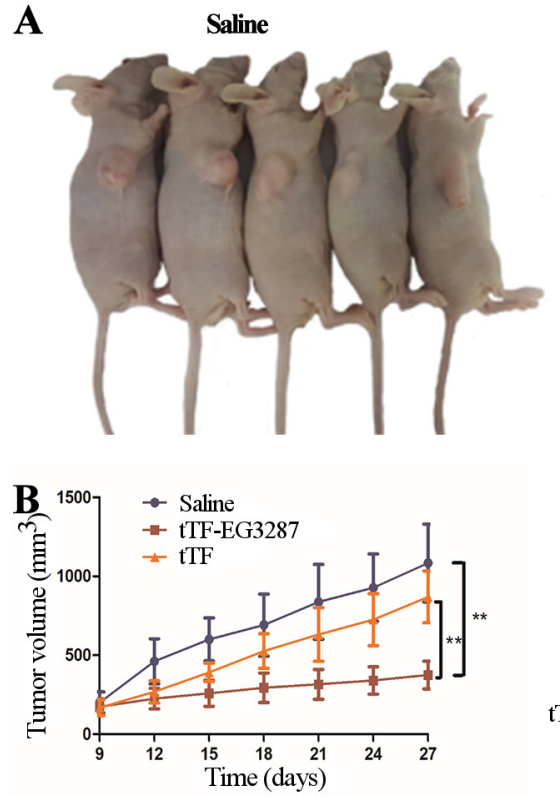

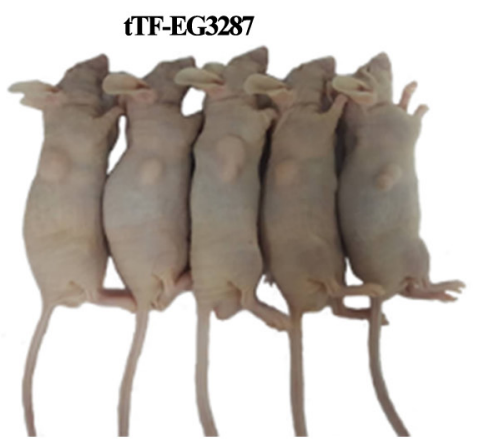

C

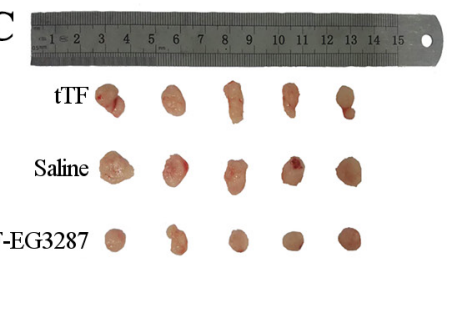

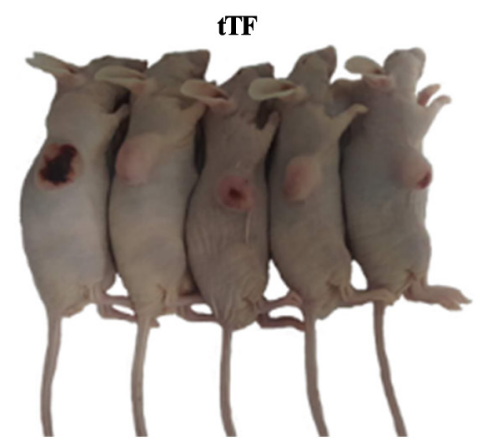

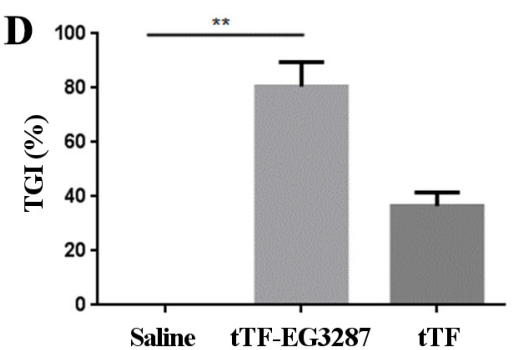

Figure 5. Therapeutic potential of tTF-EG3287 fusion protein in vivo. Nude mice with successful subcutaneous transplant were injected with tTFEG3287 $(n=5)$ and tTF ( $n=5)$ via tail vein. After 27 days, the anti-tumor effect of tTF-EG3287 was detected. A) The growth of xenografts in each group was observed after 27 days of administration. B) The growth curve of colorectal cancer xenografts in each group. C) Comparison of tumor volume and tumor suppressor rates in different groups. D) The tumor inhibition rate of tTF and tTF-EG3287. Statistical significance of differences was tested by the Mann-Whitney rank-sum test for independent groups. $\mathrm{p}<0.01$ was considered significant. 

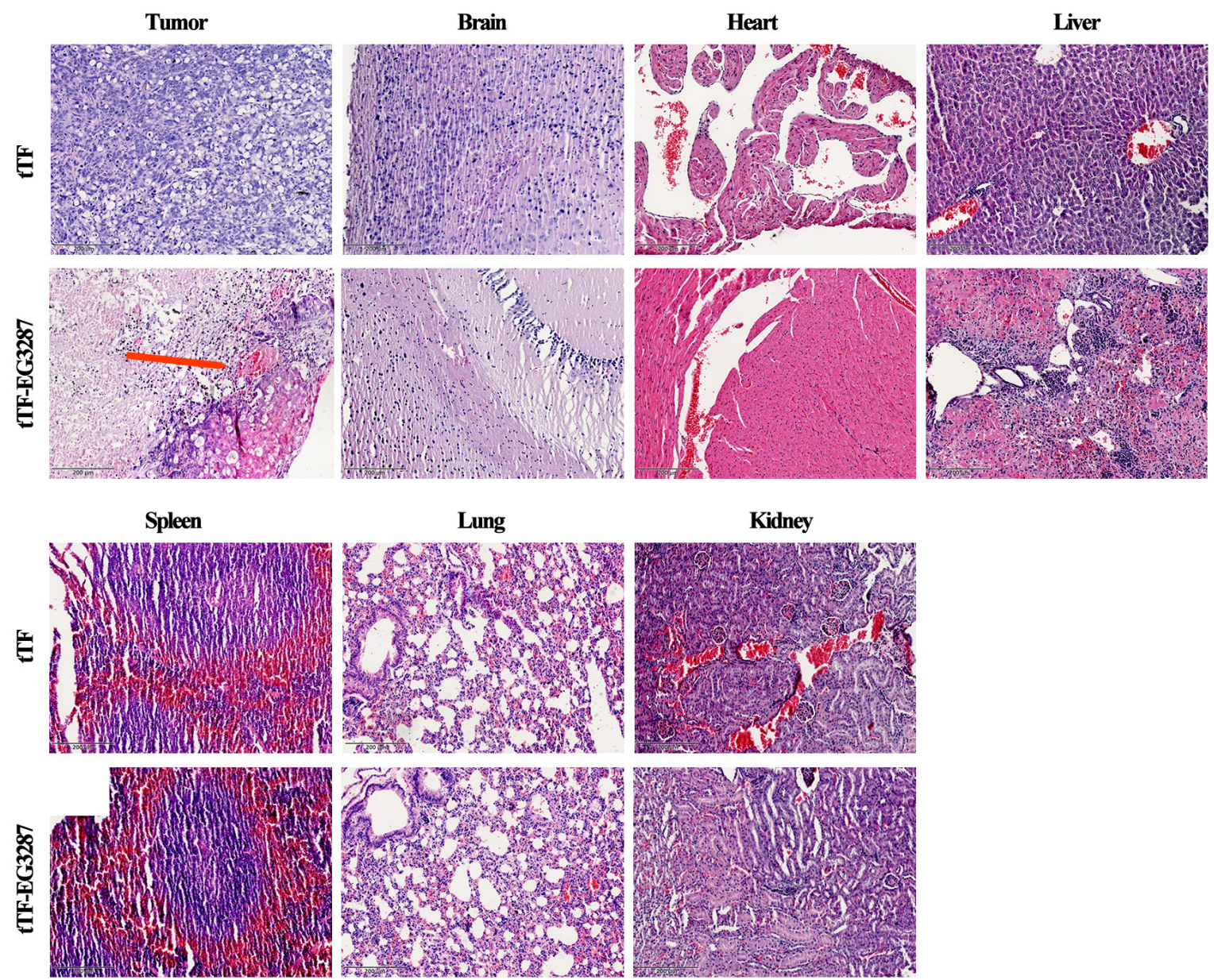

Figure 6. Effect of tTF-EG3287 on tumor thrombosis. H\&E staining of xenografts and organs of tTF or tTF-EG3287 treated HT29-bearing nude mice. Magnification: $\times 100$. Arrow: mixed thrombus within the tumor.

and liver. In lungs, due to dense capillary, large surface of endothelial cells and high relative concentration of drugs, the formation of micro-thrombus caused airway/blood flow exchange disorders and lung injury. In liver, hepatic sinus endothelial cell group with a slow blood flow is the center of substance exchange and drug metabolism, which might be the main factor for causing the toxic side effects in liver. Therefore, we need to further research the related mechanisms and lessen the side effect.

In conclusion, we optimized the induction condition and increased the amount of tTF-EG3287 fusion protein. Besides, we proved that tTF-EG3287 showed a higher pro-coagulant activity than tTF, and tTF-EG3287 was mainly located on the membrane of colon cancer cells and distributed in tumor tissues. Moreover, we also proved the anti-tumor effect of tTF-EG3287 in the treatment of colon cancer through inducing tumor thrombosis. These data provide evidence that tTF-EG3287 could be used as a novel drug for vascular targeted embolization in colon cancer.
Supplementary information is available in the online version of the paper.

Acknowledgements: The Tumor Treatment Center Special Fund and The Medical Science Innovation Fund of the Nanjing Military Region of the People's Liberation Army (grant no. 14ZD33).

\section{References}

[1] Cruz JPM, Pales CGC, Kim KM, Kim YW. Adjuvant chemotherapy for high-risk stage II and stage III colon cancer: timing of initiation and optimal duration. J BUON 2018; 23: 568-573.

[2] TSAI Y1, HUANG SC, LIN HH, LIN CC, LAN YT et al. Differences in gene mutations according to gender among patients with colorectal cancer. World J Surg Oncol 2018;16: 128. https://doi.org/10.1186/s12957-018-1431-5

[3] FRANSGAARD T, PINAR I, THYGESEN LC, GOGENUR I. Association between robot-assisted surgery and resection quality in patients with colorectal cancer. Surg Oncol 2018; 27: 177-184. https://doi.org/10.1016/j.suronc.2018.03.00 
[4] ITATANI Y, KAWADA K, SAKAI Y. Treatment of Elderly Patients with Colorectal Cancer. Biomed Res Int 2018; 2018: 2176056. https://doi.org/10.1155/2018/2176056

[5] SORBYE H, DRAGOMIR A, SUNDSTROM M, PFEIFFER P, THUNBERG $U$ et al. High BRAF Mutation Frequency and Marked Survival Differences in Subgroups According to KRAS/BRAF Mutation Status and Tumor Tissue Availability in a Prospective Population-Based Metastatic Colorectal Cancer Cohort. PLoS One 2015; 10: e0131046. https://doi. org/10.1371/journal.pone.0131046

[6] BUTT AS, NISAR N, MUGHAL T. A review: Therapeutics potentials of phytochemical drugs and their loading in $\mathrm{pH}$ specific degradable Nano-drug carrier targeting colorectal cancer. J Pak Med Assoc 2018; 68: 607-614.

[7] ZINOVIEVA OL, GRINEVA EN, PROKOFJEVA MM, KARPOV DS, ZHELTUKHIN AO et al. Expression of long non-coding RNA LINC00973 is consistently increased upon treatment of colon cancer cells with different chemotherapeutic drugs. Biochimie 2018; 151: 67-72. https://doi. org/10.1016/j.biochi.2018.05.021

[8] MARACLE CX, JEUCKEN KCM, HELDER B, VAN GULIK TM, STEINS A et al. Silencing NIK potentiates antiVEGF therapy in a novel 3D model of colorectal cancer angiogenesis. Oncotarget 2018; 9: 28445-28455. https://doi. org/10.18632/oncotarget.25442

[9] ZHANG CJ, ZHANG SY, ZHANG CD, LIN CR, LI XY et al. Usefulness of bevacizumab-induced hypertension in patients with metastatic colorectal cancer: an updated metaanalysis. Aging (Albany NY) 2018;10: 1424-1441. https:// doi.org/10.18632/aging.101478

[10] HARTY G, JARRETT J, JOFRE-BONET M. Consequences of Biomarker Analysis on the Cost-Effectiveness of Cetuximab in Combination with FOLFIRI as a First-Line Treatment of Metastatic Colorectal Cancer: Personalised Medicine at Work. Appl Health Econ Health Policy 2018; 16: 515-525. https://doi.org/10.1007/s40258-018-0395-5

[11] LV S, YE M, WANG X, LI Z, CHEN X et al. A recombined fusion protein SP5.2/tTF induce thrombosis in tumor blood vessel. Neoplasma 2015; 62: 531-540. https://doi. org/10.4149/neo_2015_064

[12] LU X1, SUN J, LIU Y, SHAO J, MA L et al. Decorating tetrathiafulvalene (TTF) with fluorinated phenyls through sulfur bridges: facile synthesis, properties, and aggregation through fluorine interactions. Chemistry 2014; 20: 96509656. https://doi.org/10.1002/chem.201402327

[13] KESSLER T1, BIEKER R, PADRO T, SCHWOPPE C, PERSIGEHL $\mathrm{T}$ et al. Inhibition of tumor growth by RGD peptide-directed delivery of truncated tissue factor to the tumor vasculature. Clin Cancer Res 2005;11: 6317-6324. https:// doi.org/10.1158/1078-0432.CCR-05-0389

[14] DREISCHALUCK J, SCHWOPPE C, SPIEKER T, KESSLER $\mathrm{T}$, TIEMANN K et al. Vascular infarction by subcutaneous application of tissue factor targeted to tumor vessels with NGR-peptides: activity and toxicity profile. Int J Oncol 2010; 37: 1389-1397. https://doi.org/10.3892/ijo_00000790

[15] JIA G, HAN Y, AN Y, DING Y, HE C et al. NRP-1 targeted and cargo-loaded exosomes facilitate simultaneous imaging and therapy of glioma in vitro and in vivo. Biomaterials 2018; 178: 302-316. https://doi.org/10.1016/j.biomaterials.2018.06.029
[16] KIM YJ, JUNG K, BAEK DS, HONG SS, KIM YS. Co-targeting of EGF receptor and neuropilin-1 overcomes cetuximab resistance in pancreatic ductal adenocarcinoma with integrin $\beta 1$-driven Src-Akt bypass signaling. Oncogene 2017; 36: 2543-2552. https://doi.org/10.1038/onc.2016.407

[17] TOMIDA C, YAMAGISHI N, NAGANO H, UCHIDA T, OHNO A et al. VEGF pathway-targeting drugs induce evasive adaptation by activation of neuropilin-1/cMet in colon cancer cells. Int J Oncol 2018; 52: 1350-1362. https://doi. org/10.3892/ijo.2018.4291

[18] JIA H, BAGHERZADEH A, HARTZOULAKIS B, JARVIS A, LOHR $M$ et al. Characterization of a bicyclic peptide neuropilin-1 (NP-1) antagonist (EG3287) reveals importance of vascular endothelial growth factor exon 8 for NP-1 binding and role of NP-1 in KDR signaling. J Biol Chem 2006; 281: 13493-13502. https://doi.org/10.1074/jbc.M512121200

[19] PARIKH AA, FAN F, LIU WB, AHMAD SA, STOELTZING O et al. Neuropilin-1 in human colon cancer: expression, regulation, and role in induction of angiogenesis. Am J Pathol 2004; 164: 2139-2151. https://doi.org/10.1016/S00029440(10)63772-8

[20] WANG L, ZENG H, WANG P, SOKER S, MUKHOPADHYAY D. Neuropilin-1-mediated vascular permeability factor/ vascular endothelial growth factor-dependent endothelial cell migration. J Biol Chem 2003; 278: 48848-48860. https:// doi.org/10.1074/jbc.M310047200

[21] UESAWA Y. [Adverse Effect Predictions Based on Computational Toxicology Techniques and Large-scale Databases]. Yakugaku Zasshi 2018; 138: 185-190. https://doi. org/10.1248/yakushi.17-00174-4

[22] XIA Y, FANG M, DONG J, XU C, LIAO Z et al. pH sensitive liposomes delivering tariquidar and doxorubicin to overcome multidrug resistance of resistant ovarian cancer cells. Colloids Surf B Biointerfaces 2018; 170: 514-520. https://doi. org/10.1016/j.colsurfb.2018.06.055

[23] RIEDEL T, CAVIN S, VAN DEN BERGH H, KRUEGER T, LIAUDET L et al. Chemo-manipulation of tumor blood vessels by a metal-based anticancer complex enhances antitumor therapy. Sci Rep 2018; 8: 10263. https://doi.org/10.1038/ s41598-018-28589-2

[24] LIS, TIANY,ZHAO Y,ZHANG Y, SUS et al.pHLIP-mediated targeting of truncated tissue factor to tumor vessels causes vascular occlusion and impairs tumor growth. Oncotarget 2015; 6: 23523-23532. https://doi.org/10.18632/oncotarget.4395

[25] BRAND C, FROHLICH M, RING J, SCHLIEMANN C, KESSLER T et al. Tumor Growth Inhibition via Occlusion of Tumor Vasculature Induced by N-Terminally PEGylated Retargeted Tissue Factor tTF-NGR. Mol Pharm 2015; 12: 3749_ 3758. https://doi.org/10.1021/acs.molpharmaceut.5b00508

[26] FANTIN A, LAMPROPOULOU A, SENATORE V, BRASH JT, PRAHST C et al. VEGF165-induced vascular permeability requires NRP1 for ABL-mediated SRC family kinase activation. J Exp Med 2017; 214: 1049-1064. https://doi. org/10.1084/jem.20160311

[27] ZHOU R, CURRY JM, ROY LD, GROVER P, HAIDER J et al. A novel association of neuropilin-1 and MUC1 in pancreatic ductal adenocarcinoma: role in induction of VEGF signaling and angiogenesis. Oncogene 2016; 35: 5608-5618. https://doi.org/10.1038/onc.2015.516 